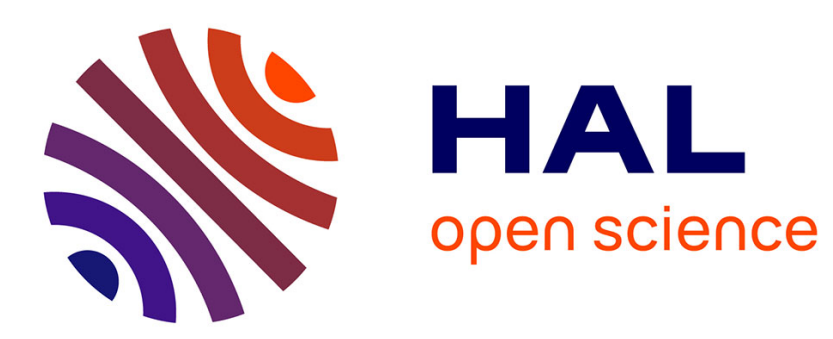

\title{
Nonlinear Impulsive Systems: 2D Stability Analysis Approach
}

Héctor Ríos, Laurentiu Hetel, Denis Efimov

\section{To cite this version:}

Héctor Ríos, Laurentiu Hetel, Denis Efimov. Nonlinear Impulsive Systems: 2D Stability Analysis Approach. Automatica, 2017, 80, pp.32-40. 10.1016/j.automatica.2017.01.010 . hal-01437308

\section{HAL Id: hal-01437308 \\ https://inria.hal.science/hal-01437308}

Submitted on 17 Jan 2017

HAL is a multi-disciplinary open access archive for the deposit and dissemination of scientific research documents, whether they are published or not. The documents may come from teaching and research institutions in France or abroad, or from public or private research centers.
L'archive ouverte pluridisciplinaire HAL, est destinée au dépôt et à la diffusion de documents scientifiques de niveau recherche, publiés ou non, émanant des établissements d'enseignement et de recherche français ou étrangers, des laboratoires publics ou privés. 


\title{
Nonlinear Impulsive Systems: 2D Stability Analysis Approach *
}

\author{
Héctor Ríos ${ }^{\mathrm{a}}$, Laurentiu Hetel ${ }^{\mathrm{b}}$ and Denis Efimov ${ }^{\mathrm{b}, \mathrm{c}}$ \\ ${ }^{a}$ CONACYT - Instituto Tecnológico de la Laguna, División de Estudios de Posgrado e Investigación, Blvd. Revolución y \\ Cuauhtémoc S/N, C.P. 27000, Torreón, Coahuila, México. \\ ${ }^{\mathrm{b}}$ CRIStAL (UMR CNRS 9189), Ecole Centrale de Lille, BP 48, Cité Scientifique, 59651 Villeneuve-d'Ascq, France. \\ ${ }^{\mathrm{c}}$ Non-A team @ Inria, Parc Scientifique de la Haute Borne, 40 avenue Halley, 59650 Villeneuve d'Ascq, France; and \\ Department of Control Systems and Informatics, Saint Petersburg State University of Information Technologies Mechanics \\ and Optics (ITMO), Kronverkskiy av. 49, Saint Petersburg, 197101, Russia.
}

\begin{abstract}
This paper contributes to the stability analysis for nonlinear impulsive dynamical systems based on a vector Lyapunov function and its divergence operator. The new method relies on a $2 D$ time domain representation. Different types of stability notions for a class of nonlinear impulsive systems are studied using a vector Lyapunov function approach. The results are applied to analyze the stability of a class of Lipschitz nonlinear impulsive systems based on Linear Matrix Inequalities. Some numerical examples illustrate the feasibility of the proposed approach.
\end{abstract}

Key words: Nonlinear impulsive systems, Stability, 2D systems.

\section{Introduction}

An important class of hybrid systems [9] is represented by impulsive systems [2]. This class of systems contain discontinuities or jumps in the state trajectories of the system governed by discrete dynamics. The dynamics of a very large variety of phenomena may be described by abrupt changes in the system state at certain instants, e.g. sample-data systems, power electronics, some models in economics, bursting rhythm models in medicine, etc. In accordance with the manner of impulses to be triggered, several kind of impulsive systems can be established: time-dependent impulsive systems, state-dependent impulsive systems, usually called reset systems, and the combination of both of them (see, e.g.

\footnotetext{
ऋ H. Ríos gratefully acknowledges the financial support from CONACyT 270504. This work was supported in part by Conseil Region Nord-Pas de Calais (ESTIREZ), the Government of Russian Federation (Grant 074-U01), the Ministry of Education and Science of Russian Federation (Project 14.Z50.31.0031), and ANR ROCC-SYS (ANR-14CE27-0008).
}

Email addresses: hriosb@correo.itlalaguna.edu.mx (Héctor Ríos), laurentiu.hetel@ec-lille.fr (Laurentiu Hetel), denis.efimov@inria.fr (Denis Efimov).
[2], [9] and [23]). In this work impulsive systems with time-triggered impulses are studied.

In the stability analysis framework of impulsive systems an important effort has been made for linear dynamics. A functional-based approach is developed for stability analysis in [6]. Some dwell-time results are established by means of this method which introduces looped functional leading to Linear Matrix Inequality (LMI) conditions. In [14], exponential stability is proposed based on Lyapunov functions with discontinuity at the impulses for nonlinear time-varying impulsive systems. These stability conditions are mainly applied to the linear case. In the sample-data system context, there exist several constructive works where the impulsive systems are used to describe the behavior of aperiodic sample-data systems (see, e.g. [10] and [19]). In [18], an equivalent correspondence between continuous and discrete time domains is provided.

With respect to the nonlinear impulsive systems, in [21] the exponential stability and stabilization problems are considered for nonlinear impulsive switched systems with time-varying disturbances. By means of switched Lyapunov function, sufficient conditions expressed as LMIs are obtained. Recently, in [12] a finite-time stabil- 
ity criteria has been established for nonlinear impulsive systems based on average impulse intervals. Nevertheless, to the best of our knowledge, the works dedicated to the stability conditions for nonlinear impulsive systems are still few. In this paper, since hybrid systems are inherently related to $2 D$ times due to the continuous and discrete variables [9], based on a $2 D$ time domain equivalence (see e.g. [17] and [22]), different types of stability notions for a class of nonlinear impulsive systems are studied using a vector Lyapunov function approach [11]. It is worth mentioning that there exist some works related to the stability for nonlinear $2 D$ discrete systems based on Lyapunov function approach (see [24] and [25]), and for nonlinear $2 D$ systems based on vector Lyapunov function approach (see [7] and [8]). However, in the last two results the boundary condition is known and decreasing. In the context of impulsive systems this means that the impulsive effects introduce stability to the system a priori. In the current work, contrarily to [7] and [8]; some conditions are provided to ensure stability and convergence for impulsive systems. Moreover, note that the results given by [7] and [8] are not directly applicable for impulsive systems since only repetitive processes are considered there and the system trajectories do not present any type of jump or discontinuity. Recently, in a previous work [16], the $2 D$ time approach was introduced to analyze the exponential stability of linear impulsive systems based on LMIs. However, only quadratic vector Lyapunov functions were considered as well as linear impulsive systems.

The main result of this work contributes to the development of a new stability analysis method for nonlinear impulsive dynamical systems based on a divergence operator of a vector Lyapunov function in a $2 \mathrm{D}$ time domain. Different types of stability notions for a class of nonlinear impulsive systems are studied using a vector Lyapunov function approach. The results are applied to analyze the asymptotic stability of a class of Lipschitz nonlinear impulsive system based on LMIs. Some numerical results illustrate the feasibility of the proposed approach.

The structure of this paper is as follows. The problem statement and preliminary definitions of different types of stability are given in Section 2. The main results as well as the stability conditions are described in Section 3. An LMI Approach for certain class of Lipschitz nonlinear impulsive systems is given in Section 4. A couple of simulation examples are considered in Section 5. Finally, some concluding remarks are discussed in Section 6.

\section{Problem statement}

Consider the class of impulsive dynamical systems for which the impulse times are external to the system and time-dependent, i.e.

$$
\begin{aligned}
& \dot{x}(t)=f_{1}(x(t)), \quad \forall t \in \mathbb{R}_{+} \backslash \mathbb{I}, \\
& x(t)=f_{2}\left(x\left(t^{-}\right)\right), \quad \forall t \in \mathbb{I}, x(0)=x_{0}
\end{aligned}
$$

where $x, x_{0} \in \mathbb{R}^{n}$ are the state of the system and the initial condition, respectively. The set of impulse times $\mathbb{I}:=\left\{t_{i}\right\}_{i \in \mathbb{N}}$ is a countable subset of $\mathbb{R}_{+}$with $t_{i+1}-t_{i}>$ 0 , for all $i \in \mathbb{N}$ and $t_{0}=0$ and $\lim _{i \rightarrow \infty} t_{i}=+\infty$ in order to avoid any zeno phenomena. The state trajectory is assumed to be right continuous and to have left limits at all times. The notation $x\left(t_{i+1}^{-}\right)=\lim _{t \uparrow t_{i+1}} x(t)$ denotes the left limit of $x(t)$ as $t$ goes to $t_{i+1}$ from the left. The distance between the impulses, i.e. the dwelltime, is defined as $T_{i}:=t_{i+1}-t_{i}$, and it is assumed that any sequence of impulse instants $\left\{t_{i}\right\}_{i \in \mathbb{N}}$ belongs to an interval, i.e. $T_{i} \in\left[T_{\min }, T_{\max }\right]$, for all $i \in \mathbb{N}$, where $T_{\min }$ and $T_{\max }$ are the minimum and maximum dwell-time, respectively. The nonlinear functions $f_{1}$ and $f_{2}$ are such that $f_{1}(0)=0$ and $f_{2}(0)=0$. It is assumed that for all $x_{0} \in \mathbb{R}^{n}, f_{1}$ is such that system (1) has a unique forward solution over any time interval $\left[t_{i}, t_{i+1}\right)$ for all $i \in \mathbb{N}$ while $f_{2}$ is a locally bounded function. In this sense, a sufficient condition for the existence of a forward solution over $\left[t_{i}, t_{i+1}\right)$ is that $f_{1}$ is Lipschitz over the same time interval.

The proposed Vector Lyapunov function based approach relies on the embedding of system (1)-(2) into a $2 D$ time domain. Indeed, the entire state trajectory $x(t)$ can be viewed as a sequence of the diagonal dynamics ${ }^{1}$ of the following $2 D$ system:

$$
\begin{aligned}
\frac{d x_{k}^{t}}{d t} & =f_{1}\left(x_{k}^{t}\right), \forall t \in\left[t_{i}, t_{i+1}\right), t_{0}=0, \\
x_{k+1}^{t} & =f_{2}\left(x_{k}^{t}\right), \forall t=t_{i+1}, \forall i=k \in \mathbb{N}, x_{0}^{0}=x_{0},
\end{aligned}
$$

where $x_{k}^{t}:\left[t_{i}, t_{i+1}\right) \times \mathbb{N} \rightarrow \mathbb{R}^{n}$ is the current state vector, $x_{k+1}^{t_{i+1}}: t_{i+1} \times \mathbb{N} \rightarrow \mathbb{R}^{n}$ represents the reset vector state, $x_{k}^{t_{i+1}}: t_{i+1} \times \mathbb{N} \rightarrow \mathbb{R}^{n}$ denotes the value of $x$ just before the impulse $k+1$, for all $i=k \in \mathbb{N}$, i.e. $x_{k}^{t_{i+1}}=\lim _{t \uparrow t_{i+1}} x_{k}^{t}$, for $t<t_{i+1}$. It is worth saying that the solutions of system (3) are unique for the diagonal dynamics, i.e. for all $i=k \in \mathbb{N}, t \in \mathbb{R}_{+}$; and they are represented by a sequence of functions $\left\{x_{k}^{t_{i}+\tau}, \tau \in\left(0, t_{i+1}\right]\right\}_{i}$, for all $i=k \in \mathbb{N}$. Note that the discrete time $k$ depicts the number of impulses on the system.

The goal in this work is to find the conditions for the stability and asymptotic stability of the impulsive systems (1)-(2) by means of the $2 D$ time representation (3)-(4).

\footnotetext{
1 The diagonal dynamics make reference only to those dynamics given by (3)-(4) corresponding to $i=k$, for all $i, k \in \mathbb{N}$ and for all $t \in \mathbb{R}_{+}$.
} 


\subsection{Preliminaries}

A continuous function $\sigma: \mathbb{R}_{+} \rightarrow \mathbb{R}_{+}$belongs to class $\mathcal{K}$ if it is strictly increasing and $\sigma(0)=0$; it belongs to class $\mathcal{K}_{\infty}$ if it is also unbounded. A continuous function $\beta: \mathbb{R}_{+} \times \mathbb{R}_{+} \rightarrow \mathbb{R}_{+}$belongs to class $\mathcal{K} \mathcal{L}$ if, for each fixed $s, \beta(r, s) \in \mathcal{K}$ with respect to $r$ and, for each fixed $r$, $\beta(r, s)$ is decreasing with respect to $s$ and $\beta(r, s) \rightarrow 0$ as $s \rightarrow \infty$. Let $|q|$ denote the Euclidean norm of a vector $q$. The symbols $\wedge$ and $\vee$ denote the logic operations "and" and "or", respectively.

Let $\Omega$ be an open subset of $\mathbb{R}^{n}$, such that $0 \in \Omega$. The following stability definitions are introduced:

Definition 1 A 2D system described by (3)-(4) is said to be Diagonal Stable (DS) in $\Omega$, if there exist functions $\sigma_{1}, \sigma_{2} \in \mathcal{K}_{\infty}$ and some constants $\kappa_{1}, \kappa_{2}>0$ such that, for all $i=k \in \mathbb{N}$

$$
\begin{gathered}
\left|x_{k+1}^{t_{i+1}}\right| \leq \sigma_{1}\left(\left|x_{0}\right|\right)+\kappa_{1}, \forall x_{0} \in \Omega, \\
\left|x_{k}^{t}\right| \leq \sigma_{2}\left(\left|x_{k}^{t_{i}}\right|\right)+\kappa_{2}, \forall t \in\left[t_{i}, t_{i+1}\right) .
\end{gathered}
$$

Definition 2 A 2D system described by (3)-(4) is said to be Asymptotically Diagonal Stable $(A D S)$ in $\Omega$, if there exist functions $\beta \in \mathcal{K} \mathcal{L}$ and $\sigma \in \mathcal{K}_{\infty}$ such that, for all $i=k \in \mathbb{N}$

$$
\begin{gathered}
\left|x_{k+1}^{t_{i+1}}\right| \leq \beta\left(\left|x_{0}\right|, k\right), \forall x_{0} \in \Omega, \\
\left|x_{k}^{t}\right| \leq \sigma\left(\left|x_{k}^{t_{i}}\right|\right), \forall t \in\left[t_{i}, t_{i+1}\right) .
\end{gathered}
$$

Definition 3 A 2D system described by (3)-(4) is said to be Strongly Asymptotically Diagonal Stable (SADS) in $\Omega$, if there exist functions $\beta_{1}, \beta_{2} \in \mathcal{K} \mathcal{L}$ such that, for all $i=k \in \mathbb{N}$

$$
\begin{gathered}
\left|x_{k+1}^{t_{i+1}}\right| \leq \beta_{1}\left(\left|x_{0}\right|, k\right), \quad \forall x_{0} \in \Omega, \\
\left|x_{k}^{t}\right| \leq \beta_{2}\left(\left|x_{k}^{t_{i}}\right|, t-t_{i}\right), \quad \forall t \in\left[t_{i}, t_{i+1}\right) .
\end{gathered}
$$

If $\Omega=\mathbb{R}^{n}$, then the $2 \mathrm{D}$ system is said to be globally DS $(G D S), \operatorname{ADS}(G A D S)$, or SADS $(G S A D S)$, respectively. Note that the Definition 3, i.e. strong asymptotical diagonal stability; is a particular case of the Definition 2, i.e. asymptotical diagonal stability.

\section{$3 \quad$ Stability analysis}

In order to give the stability conditions a vector Lyapunov approach is used [16]:

$$
V\left(t, x_{k}^{t}, x_{k+1}^{t_{i+1}}\right)=\left(\begin{array}{c}
V_{1}\left(t, x_{k}^{t}\right) \\
V_{2}\left(x_{k+1}^{t_{i+1}}\right)
\end{array}\right),
$$

where $V_{1}(t, x)>0, V_{2}(x)>0$, for all $t \geq 0$ and $x \neq 0$, and $V_{1}(t, 0)=0, V_{2}(0)=0$, for all $t \geq 0$. Now, the divergence operator of the candidate function $V$ along the trajectories of system (3)-(4) is introduced for all $t \in\left[t_{i}, t_{i+1}\right):$

$$
\operatorname{div} V(\cdot)=\frac{d V_{1}\left(t, x_{k}^{t}\right)}{d t}+V_{2}\left(x_{k+1}^{t_{i+1}}\right)-V_{2}\left(x_{k}^{t_{i+1}}\right),
$$

i.e. $V_{1}$ is differentiable with respect to the continuous time $t$ while the difference in $V_{2}$ is calculated in the discrete time $k$.

\subsection{Minimum dwell-time results}

Let $\Omega$ be an open neighborhood of the origin. Then, based on the vector Lyapunov function and its divergence, the following theorem is established: ${ }^{2}$

Theorem 1 (Diagonal stability - Minimum dwelltime). Assume that there exist positive constants $\varepsilon \in(0,1), a_{1}, a_{2}, b_{1}, b_{2}, c_{1}, c_{2}, c_{3}, c_{4}$ and $c_{5}$ such that the vector Lyapunov function $V(\cdot)$ and its divergence along the trajectories of the system (3)-(4) satisfy the following inequalities for all $i=k \in \mathbb{N}, x_{k}^{t} \in \Omega$ and $t \in\left[t_{i}, t_{i+1}\right)$

$$
\begin{gathered}
c_{1}\left|x_{k}^{t}\right|^{a_{1}} \leq V_{1}\left(t, x_{k}^{t}\right) \leq c_{2}\left|x_{k}^{t}\right|^{a_{2}}, \\
c_{3}\left|x_{k}^{p}\right|^{b_{1}} \leq V_{2}\left(x_{k}^{p}\right) \leq c_{4}\left|x_{k}^{p}\right|^{b_{2}}, \forall p=t_{i}, t_{i+1}, \\
\operatorname{div} V \leq-c_{5}\left(\left|x_{k}^{t}\right|^{a_{2}}+\left|x_{k}^{t_{i+1}}\right|^{b_{2}}\right), \\
c_{4} \leq c_{5}, \\
b_{1}>a_{2} \wedge T_{\min }=\frac{c_{2}}{c_{5}} \gamma<T_{i},
\end{gathered}
$$

where $\gamma=-\ln \left[c_{3}^{a_{2} / b_{1}}(1-\varepsilon) /\left(c_{5}+c_{3}^{a_{2} / b_{1}}(1-\varepsilon)\right)\right]$. Then, the $2 D$ system (3)-(4) is DS in $\Omega$ for any sequence $T_{i} \in$ $\left[T_{\min }, \infty\right)$.

Proof. From the divergence definition and inequalities (13), (14) and (15), it follows that

$$
\begin{aligned}
\frac{d V_{1}\left(t, x_{k}^{t}\right)}{d t} & \leq-c_{5}\left(\left|x_{k}^{t}\right|^{a_{2}}+\left|x_{k}^{t_{i+1}}\right|^{b_{2}}\right)-V_{2}\left(x_{k+1}^{t_{i+1}}\right)+V_{2}\left(x_{k}^{t_{i+1}}\right) \\
& \leq-\beta V_{1}\left(t, x_{k}^{t}\right)+\lambda V_{2}\left(x_{k}^{t_{i+1}}\right)-V_{2}\left(x_{k+1}^{t_{i+1}}\right)
\end{aligned}
$$

where $\lambda=\left(c_{4}-c_{5}\right) / c_{4}$ and $\beta=c_{5} / c_{2}$. By means of the comparison principle, with respect to the time $t$, from (18), for all $t \in\left[t_{i}, t_{i+1}\right)$, it is obtained that

$$
\begin{aligned}
V_{1}\left(t, x_{k}^{t}\right) \leq & e^{-\beta\left(t-t_{i}\right)} V_{1}\left(t_{i}, x_{k}^{t_{i}}\right)+\int_{t_{i}}^{t} e^{-\beta(t-\tau)} \\
& \times\left[\lambda V_{2}\left(x_{k}^{t_{i+1}}\right)-V_{2}\left(x_{k+1}^{t_{i+1}}\right)\right] d \tau \\
& =e^{-\beta\left(t-t_{i}\right)} V_{1}\left(t_{i}, x_{k}^{t_{i}}\right) \\
& +\rho_{i}(t)\left[\lambda V_{2}\left(x_{k}^{t_{i+1}}\right)-V_{2}\left(x_{k+1}^{t_{i+1}}\right)\right]
\end{aligned}
$$

\footnotetext{
2 It is worth mentioning that the results provided in this work generalize those given in [16], for a larger class of systems.
} 
where $\rho_{i}(t)=\left(1-e^{-\beta\left(t-t_{i}\right)}\right) / \beta \geq 0$ for all $t \in\left[t_{i}, t_{i+1}\right]$. Now, the proof is split into two parts: convergence in the impulse times, and boundedness between the impulses, i.e. eqs. (5) and (6) from Definition 1, respectively.

a) Convergence in the impulse times. Evaluating (19) in $t=t_{i+1}$, it gives

$$
\begin{aligned}
& V_{1}\left(t_{i+1}, x_{k}^{t_{i+1}}\right) \leq e^{-\beta T_{i}} V_{1}\left(t_{i}, x_{k}^{t_{i}}\right) \\
& \quad+\rho_{i}\left(t_{i+1}\right) \lambda V_{2}\left(x_{k}^{t_{i+1}}\right)-\rho_{i}\left(t_{i+1}\right) V_{2}\left(x_{k+1}^{t_{i+1}}\right) .
\end{aligned}
$$

From the inequalities (13) and (14), it follows that for all $i=k \in \mathbb{N}$

$$
\begin{aligned}
& \frac{V_{2}^{\alpha_{1}}\left(x_{k}^{p}\right)}{c_{4}^{\alpha_{1}}} \leq\left|x_{k}^{p}\right|^{a_{1}} \leq \frac{V_{1}\left(p, x_{k}^{p}\right)}{c_{1}}, \forall p=t_{i}, t_{i+1}, \\
& \frac{V_{1}^{\beta_{1}}\left(p, x_{k}^{p}\right)}{c_{2}^{\beta_{1}}} \leq\left|x_{k}^{p}\right|^{b_{1}} \leq \frac{V_{2}\left(x_{k}^{p}\right)}{c_{3}}, \forall p=t_{i}, t_{i+1},
\end{aligned}
$$

where $\alpha_{1}=a_{1} / b_{2}$ and $\beta_{1}=b_{1} / a_{2}$. From (21), it is given that $c_{1} c_{4}^{-\alpha_{1}} V_{2}^{\alpha_{1}}\left(x_{k}^{t_{i+1}}\right) \leq V_{1}\left(t_{i+1}, x_{k}^{t_{i+1}}\right)$, and therefore from (20), it is obtained that

$$
\begin{aligned}
\rho_{i}\left(t_{i+1}\right) & V_{2}\left(x_{k+1}^{t_{i+1}}\right) \leq e^{-\beta T_{i}} V_{1}\left(t_{i}, x_{k}^{t_{i}}\right) \\
& +\rho_{i}\left(t_{i+1}\right) \lambda V_{2}\left(x_{k}^{t_{i+1}}\right)-c_{1} c_{4}^{-\alpha_{1}} V_{2}^{\alpha_{1}}\left(x_{k}^{t_{i+1}}\right) .
\end{aligned}
$$

Consider that $c_{5} \geq c_{4}$, i.e. $\lambda \leq 0$ and (16) holds; using (22) and (23) it follows that

$$
V_{2}\left(x_{k+1}^{t_{i+1}}\right) \leq \varphi_{i} V_{2}^{1 / \beta_{1}}\left(x_{k}^{t_{i}}\right),
$$

where $\varphi_{i}=c_{2} e^{-\beta T_{i}} / c_{3}^{1 / \beta_{1}} \rho_{i}\left(t_{i+1}\right)$; and then, by induction, it is obtained that

$$
V_{2}\left(x_{k+1}^{t_{i+1}}\right) \leq \varphi_{i}^{p(k)} V_{2}^{\left(1 / \beta_{1}\right)^{k}}\left(x_{0}\right),
$$

where $p(k)=\sum_{j=0}^{k}\left(1 / \beta_{1}\right)^{j}$. Assume that $\beta_{1}>1$, i.e. $b_{1}>a_{2}$, then $p(k)$ is a geometric series; that there exists a $\varepsilon \in(0,1)$, and that the following condition holds

$$
\varphi_{i}=\frac{c_{2} e^{-\beta T_{i}}}{c_{3}^{1 / \beta_{1}} \rho_{i}\left(t_{i+1}\right)}<1-\varepsilon \Leftrightarrow T_{\min }>\frac{c_{2}}{c_{5}} \gamma .
$$

Note that the last inequality is the same that (17) with $\gamma=-\ln \left[c_{3}^{1 / \beta_{1}}(1-\varepsilon) /\left(c_{5}+c_{3}^{1 / \beta_{1}}(1-\varepsilon)\right)\right]$. Then, taking into account that $\rho_{i}\left(t_{i+1}\right)=c_{2}\left(1-e^{-\beta T_{i}}\right) / c_{5}$, one obtains from (25) that

$$
V_{2}\left(x_{k+1}^{t_{i+1}}\right) \leq \varphi_{i}\left(V_{2}\left(x_{0}\right)+1\right) \leq \frac{c_{5}\left(V_{2}\left(x_{0}\right)+1\right)}{c_{3}^{1 / \beta_{1}}\left(1-e^{-\gamma}\right)} .
$$

Thus, using (21) and (22), it follows that for all $i=k \in \mathbb{N}$

$$
\left|x_{k+1}^{t_{i+1}}\right| \leq \sigma_{1}\left(\left|x_{0}\right|\right)+\kappa_{1}, \forall x_{0} \in \Omega,
$$

where

$$
\begin{gathered}
\sigma_{1}\left(\left|x_{0}\right|\right)=\left(\frac{c_{4} c_{5}\left|x_{0}\right|^{b_{2}}}{c_{3}^{\left(a_{2}+b_{1}\right) / b_{1}}\left(1-e^{-\gamma}\right)}\right)^{\frac{1}{b_{1}}}, \\
\kappa_{1}=\left(\frac{c_{5}}{c_{3}^{\left(a_{2}+b_{1}\right) / b_{1}}\left(1-e^{-\gamma}\right)}\right)^{\frac{1}{b_{1}}} .
\end{gathered}
$$

Therefore, it is concluded that the trajectories of system (3)-(4) are stable (Definition 1, eq. (5)), under constraints (16) and (17).

b) Boundedness between the impulses. From (19) the negative term $\rho_{i}(t) V_{2}\left(x_{k+1}^{t_{i+1}}\right)$ can be disregarded, then it is given that

$$
V_{1}\left(t, x_{k}^{t}\right) \leq e^{-\beta\left(t-t_{i}\right)} V_{1}\left(t_{i}, x_{k}^{t_{i}}\right)+\rho_{i}\left(t_{i+1}\right) \lambda V_{2}\left(x_{k}^{t_{i+1}}\right) .
$$

Consider that $c_{5} \geq c_{4}$, i.e. $\lambda \leq 0$ and (16) holds; from $(29)$, it follows that $V_{1}\left(t, x_{k}^{t}\right) \leq e^{-\beta\left(t-t_{i}\right)} V_{1}\left(t_{i}, x_{k}^{t_{i}}\right)$ and boundedness is given, i.e. $\left|x_{k}^{t}\right| \leq \sigma_{2}\left(\left|x_{k}^{t_{i}}\right|\right), \forall t \in$ $\left[t_{i}, t_{i+1}\right)$, where $\sigma_{2}\left(\left|x_{k}^{t_{i}}\right|\right)=\left(c_{2}\left|x_{k}^{t_{i}}\right|^{a_{2}} / c_{1}\right)^{1 / a_{1}}$. Thus, during each interval between impulses the trajectories of the system are bounded (Definition 1, eq. (6)), and due to the stability property given by (5), the $2 \mathrm{D}$ system described by (3)-(4) is DS in $\Omega$.

Remark 1 Note that if (26) is satisfied for all $x_{0} \in \Omega=$ $\mathbb{R}^{n}$, then one gets global diagonal stability.

Let us consider the particular case in which $b_{1}=a_{2}$, i.e. $\beta_{1}=1$. Then, the following result is obtained.

Corollary 2 (Strong asymptotic diagonal stability Minimum dwell-time). Assume that there exist positive constants $\varepsilon \in(0,1), a_{1}, a_{2}, b_{1}, b_{2}, c_{1}, c_{2}, c_{3}, c_{4}$ and $c_{5}$ such that the vector Lyapunov function $V(\cdot)$ and its divergence along the trajectories of the system (3)-(4) satisfy (13), (14), (15), (16), and the following constraints, for all $i=k \in \mathbb{N}, x_{k}^{t} \in \Omega$ and $t \in\left[t_{i}, t_{i+1}\right)$

$$
b_{1}=a_{2} \wedge T_{\min }=\frac{c_{2}}{c_{5}} \gamma<T_{i}
$$

where $\gamma=-\ln \left[c_{3}(1-\varepsilon) /\left(c_{5}+c_{3}(1-\varepsilon)\right)\right]$. Then, the $2 D$ system (3)-(4) is SADS in $\Omega$ for any sequence $T_{i} \in$ $\left[T_{\min }, \infty\right)$.

Proof. Note that (19), (20), (21), (22) and (23) hold for any possible value of $a_{1}, a_{2}, b_{1}$ and $b_{2}$. Then, recalling that $c_{5} \geq c_{4}$, i.e. $\lambda \leq 0$; from (23) it follows that for $\beta_{1}=1, V_{2}\left(x_{k+1}^{t_{i+1}}\right) \leq \varphi_{i}^{\prime} V_{2}\left(x_{k}^{t_{i}}\right)$, where $\varphi_{i}^{\prime}=\frac{c_{2} e^{-\beta T_{i}}}{c_{3} \rho_{i}\left(t_{i+1}\right)} ;$ and then, by induction, it is given

$$
V_{2}\left(x_{k+1}^{t_{i+1}}\right) \leq \varphi_{i}^{\prime k+1} V_{2}\left(x_{0}\right),
$$

which is decreasing if the condition $T_{\min }>c_{2} \gamma / c_{5}$, with $\gamma=-\ln \left[c_{3}(1-\varepsilon) /\left(c_{5}+c_{3}(1-\varepsilon)\right)\right]$; holds. Therefore, it is obtained that for all $i=k \in \mathbb{N}$

$$
\left|x_{k+1}^{t_{i+1}}\right| \leq \beta_{1}\left(\left|x_{0}\right|, k\right), \forall x_{0} \in \Omega,
$$

with $\beta_{1}\left(\left|x_{0}\right|, k\right)=c^{1 / b_{1}} \kappa_{1}^{(k+1) / b_{1}}\left|x_{0}\right|^{b_{2} / b_{1}}$, where $c=$ $c_{4} / c_{3}$, and, from the fact that $T_{\min }>c_{2} \gamma / c_{5} ; \kappa_{1}=$ $c_{5} / c_{3}\left(1-e^{-\gamma}\right)<1$. Hence, the trajectories of system (3)-(4) converge to zero in the impulse times under constraints $T_{\min }>c_{2} \gamma / c_{5}$ and $b_{1}=a_{2}$, if (16) holds. 
Note that the boundedness between the impulses can be proven in the same way as in Theorem 1 . In fact, from (19), it follows that for $c_{5} \geq c_{4}$, i.e. $\lambda \leq 0$, both negative terms $\rho_{i}(t) \lambda V_{2}\left(x_{k}^{\bar{t}_{i+1}}\right)$ and $\rho_{i}(t) V_{2}\left(x_{k+1}^{t_{i+1}}\right)$ can be disregarded, then $V_{1}\left(t, x_{k}^{t}\right) \leq$ $e^{-\beta\left(t-t_{i}\right)} V_{1}\left(t_{i}, x_{k}^{t_{i}}\right)$ holds and boundedness is provided, i.e. $\left|x_{k}^{t}\right| \leq \beta_{2}\left(\left|x_{k}^{t_{i}}\right|, t-t_{i}\right), \quad \forall t \in\left[t_{i}, t_{i+1}\right)$, where $\beta_{2}\left(\left|x_{k}^{t_{i}}\right|, t-t_{i}\right)=\left(c_{2} e^{-\beta\left(t-t_{i}\right)}\left|x_{k}^{t_{i}}\right|^{a_{2}} / c_{1}\right)^{1 / a_{1}}$. Thus, during each interval between impulses the trajectories of the system are bounded (Definition 3, eq. (10)), and due to the convergence property given by (9), the $2 \mathrm{D}$ system described by (3)-(4) is SADS in $\Omega$. Hence, the corollary has been proven.

Remark 2 As in Theorem 1, note that the results become global if (32) holds for all $x_{0} \in \Omega=\mathbb{R}^{n}$.

\subsection{Maximum or ranged dwell-time results}

Now, the following results describe the diagonal stability under maximum or ranged dwell-time conditions which come from the case in which condition (16) is not satisfied, i.e. for the case $c_{4}>c_{5}$.

Theorem 3 (Diagonal stability - Maximum/Ranged dwell-time). Assume that there exist positive constants $\varepsilon \in(0,1), a_{1}, a_{2}, b_{1}, b_{2}, c_{1}, c_{2}, c_{3}, c_{4}$ and $c_{5}$ such that the vector Lyapunov function $V(\cdot)$ and its divergence along the trajectories of the system (3)-(4) satisfy (13), (14), (15), and the following constraints, for all $i=k \in \mathbb{N}$, $x_{k}^{t} \in \Omega$ and $t \in\left[t_{i}, t_{i+1}\right)$

$$
\begin{aligned}
c_{4} & >c_{5} \wedge a_{1}>b_{2} \\
c_{2} c_{4}^{\alpha_{1}-1}\left(c_{4}-c_{5}\right) & \leq c_{1} c_{5} \vee T_{i}<\frac{c_{2}}{c_{5}} \alpha=T_{\max }, \\
b_{1}>a_{2} & \wedge T_{\min }=\frac{c_{2}}{c_{5}} \gamma<T_{i}
\end{aligned}
$$

where $\gamma=-\ln \left[c_{3}^{a_{2} / b_{1}}(1-\varepsilon) /\left(c_{5}+c_{3}^{a_{2} / b_{1}}(1-\varepsilon)\right)\right]$, and

$$
\alpha=-\ln \left(\frac{c_{2} c_{4}^{\left(a_{1}-b_{1}\right) / b_{1}}\left(c_{4}-c_{5}\right)-c_{1} c_{5}}{c_{2} c_{4}^{\left(a_{1}-b_{1}\right) / b_{1}}\left(c_{4}-c_{5}\right)}\right),
$$

for all $c_{2} c_{4}^{\left(a_{1}-b_{1}\right) / b_{1}}\left(c_{4}-c_{5}\right)>c_{1} c_{5}$. Then, the $2 D$ system (3)-(4) is $D S$ in $\Omega$ for any sequence $T_{i} \in\left[T_{\min }, T_{\max }\right]$.

Proof. From (33) it follows that $c_{4}>c_{5}$, i.e. $\lambda \in(0,1)$; and $\alpha_{1}>1$, i.e. $a_{1}>b_{2}$. Then, it is satisfied that

$$
\begin{aligned}
& V_{2}\left(x_{k}^{t_{i+1}}\right)<V_{2}^{\alpha_{1}}\left(x_{k}^{t_{i+1}}\right), \text { for } V_{2}\left(x_{k}^{t_{i+1}}\right)>1, \\
& V_{2}\left(x_{k}^{t_{i+1}}\right) \geq V_{2}^{\alpha_{1}}\left(x_{k}^{t_{i+1}}\right), \text { for } V_{2}\left(x_{k}^{t_{i+1}}\right) \leq 1 .
\end{aligned}
$$

It is worth mentioning that (19), (20), (21), (22) and (23) hold for any possible value of $a_{1}, a_{2}, b_{1}$ and $b_{2}$. Therefore, similar arguments as in the proof of Theorem 1 may be used to prove this theorem. a) Convergence in the impulse times. Let us consider two different cases for the convergence proof, i.e. $V_{2}\left(x_{k}^{t_{i+1}}\right)>1$ and $V_{2}\left(x_{k}^{t_{i+1}}\right) \leq 1$. Therefore, from (23), it follows that when $V_{2}\left(x_{k}^{t_{i+1}}\right)>1$

$$
\begin{aligned}
\rho_{i}\left(t_{i+1}\right) V_{2}\left(x_{k+1}^{t_{i+1}}\right) & \leq \frac{c_{2} e^{-\beta T_{i}}}{c_{3}^{1 / \beta_{1}}} V_{2}^{1 / \beta_{1}}\left(x_{k}^{t_{i}}\right) \\
& +\left(\rho_{i}\left(t_{i+1}\right) \lambda-c_{1} c_{4}^{-\alpha_{1}}\right) V_{2}\left(x_{k}^{t_{i+1}}\right) .
\end{aligned}
$$

Note that if the constraint $\rho_{i}\left(t_{i+1}\right) \lambda<c_{1} c_{4}^{-\alpha_{1}}$ holds, then the term depended on $V_{2}\left(x_{k}^{t_{i+1}}\right)$ can be disregarded. In this sense, in order to satisfy such a constraint, recalling that $\beta=c_{5} / c_{2}, \lambda=\left(c_{4}-c_{5}\right) / c_{4}$ and $\rho_{i}\left(t_{i+1}\right)=$ $c_{2}\left(1-e^{-\beta T_{i}}\right) / c_{5}$, the following condition is founded

$$
\begin{aligned}
c_{2} c_{4}^{\alpha_{1}-1}\left(1-e^{-\beta T_{i}}\right)\left(c_{4}-c_{5}\right) & <c_{1} c_{5}, \\
\left(1-e^{-\beta T_{i}}\right) & <\frac{c_{1} c_{5}}{c_{2} c_{4}^{\alpha_{1}-1}\left(c_{4}-c_{5}\right)} .
\end{aligned}
$$

Thus, it is clear that if $c_{2} c_{4}^{\alpha_{1}-1}\left(c_{4}-c_{5}\right) \leq c_{1} c_{5}$ holds then $\rho_{i}\left(t_{i+1}\right) \lambda<c_{1} c_{4}^{-\alpha_{1}}$ is trivially satisfied. Otherwise

$$
e^{-\beta T_{i}}>\frac{c_{2} c_{4}^{\alpha_{1}-1}\left(c_{4}-c_{5}\right)-c_{1} c_{5}}{c_{2} c_{4}^{\alpha_{1}-1}\left(c_{4}-c_{5}\right)} \Leftrightarrow T_{\max }<\frac{c_{2}}{c_{5}} \alpha,
$$

where

$$
\alpha=-\ln \left(\frac{c_{2} c_{4}^{\left(a_{1}-b_{1}\right) / b_{1}}\left(c_{4}-c_{5}\right)-c_{1} c_{5}}{c_{2} c_{4}^{\left(a_{1}-b_{1}\right) / b_{1}}\left(c_{4}-c_{5}\right)}\right)>0,
$$

for all $c_{2} c_{4}^{\alpha_{1}-1}\left(c_{4}-c_{5}\right)>c_{1} c_{5}$. Note that these two possibilities, i.e. $c_{2} c_{4}^{\alpha_{1}-1}\left(c_{4}-c_{5}\right) \leq c_{1} c_{5}$ or $T_{i}<c_{2} \alpha / c_{5}$, are represented by (34) in Theorem 3 . Therefore, if one of them is satisfied, from (36), one obtains $V_{2}\left(x_{k+1}^{t_{i+1}}\right) \leq$ $\varphi_{i} V_{2}^{1 / \beta_{1}}\left(x_{k}^{t_{i}}\right)$, and using similar arguments as in Theorem 1 , if $\beta_{1}>1$, i.e. $b_{1}>a_{2}$, and $T_{i}>c_{2} \gamma / c_{5}$, with $\gamma=-\ln \left[c_{3}^{a_{2} / b_{1}}(1-\varepsilon) /\left(c_{5}+c_{3}^{a_{2} / b_{1}}(1-\varepsilon)\right)\right]$, it follows that for all $i=k \in \mathbb{N},\left|x_{k+1}^{t_{i+1}}\right| \leq \sigma_{1}^{\prime}\left(\left|x_{0}\right|\right)+\kappa_{1}^{\prime}, \forall x_{0} \in \Omega$, for $V_{2}\left(x_{k}^{t_{i+1}}\right)>1$ with

$$
\begin{gathered}
\sigma_{1}^{\prime}\left(\left|x_{0}\right|\right)=\left(\frac{c_{4} c_{5}\left|x_{0}\right|^{b_{2}}}{c_{3}^{\left(a_{2}+b_{1}\right) / b_{1}}\left(1-e^{-\gamma}\right)}\right)^{\frac{1}{b_{1}}}, \\
\kappa_{1}^{\prime}=\left(\frac{c_{5}}{c_{3}^{\left(a_{2}+b_{1}\right) / b_{1}}\left(1-e^{-\gamma}\right)}\right)^{\frac{1}{b_{1}}} .
\end{gathered}
$$

Therefore, it is possible to conclude that when $V_{2}\left(x_{k}^{t_{i+1}}\right)$ $>1$ the trajectories of system (3)-(4) are bounded.

To complete the convergence proof let us consider the second case, i.e. $V_{2}\left(x_{k}^{t_{i+1}}\right) \leq 1$. Using (22) and dividing by $\rho_{i}\left(t_{i+1}\right)(23)$, it follows that for $V_{2}\left(x_{k}^{t_{i+1}}\right) \leq 1$, $V_{2}\left(x_{k+1}^{t_{i+1}}\right) \leq \varphi_{i} V_{2}^{1 / \beta_{1}}\left(x_{k}^{t_{i}}\right)+\lambda$. Then, by induction, it is given that 


$$
V_{2}\left(x_{k+1}^{t_{i+1}}\right) \leq \varphi_{i}^{p(k)} V_{2}^{\left(1 / \beta_{1}\right)^{k}}\left(x_{0}\right)+\sum_{l=0}^{k} \varphi_{i}^{p(l)-\left(1 / \beta_{1}\right)^{l}} \lambda^{\left(1 / \beta_{1}\right)^{l}},
$$

where $p(k)=\sum_{j=0}^{k}\left(1 / \beta_{1}\right)^{j}$ is a geometric series if $\beta_{1}>$ 1 . Then, taking into account that $\beta_{1}>1, T_{\min }>c_{2} \gamma / c_{5}$, $\rho_{i}\left(t_{i+1}\right)=c_{2}\left(1-e^{-\beta T_{i}}\right) / c_{5}$ and $\lambda \in(0,1)$; the previous inequality can be majorized by the following expression

$$
V_{2}\left(x_{k+1}^{t_{i+1}}\right) \leq \frac{c_{5}\left(V_{2}\left(x_{0}\right)+2\right)}{c_{3}^{1 / \beta_{1}}\left(1-e^{-\gamma}\right)\left(1-\lambda^{1 / \beta_{1}}\right)} .
$$

Thus, using (21) and (22), one gets that for all $i=k \in \mathbb{N}$, $\left|x_{k+1}^{t_{i+1}}\right| \leq \sigma_{1}\left(\left|x_{0}\right|\right)+\kappa_{1}, \forall x_{0} \in \Omega$, for $V_{2}\left(x_{k}^{t_{i+1}}\right) \leq 1$ with

$$
\begin{gathered}
\sigma_{1}\left(\left|x_{0}\right|\right)=\left(\frac{c_{3}^{-\left(a_{2}+b_{1}\right) / b_{1}} c_{4} c_{5}\left|x_{0}\right|^{b_{2}}}{\left(1-e^{-\gamma}\right)\left(1-\lambda^{a_{2} / b_{1}}\right)}\right)^{\frac{1}{b_{1}}}, \\
\kappa_{1}=\left(\frac{2 c_{3}^{-\left(a_{2}+b_{1}\right) / b_{1}} c_{5}}{\left(1-e^{-\gamma}\right)\left(1-\lambda^{a_{2} / b_{1}}\right)}\right)^{\frac{1}{b_{1}}} .
\end{gathered}
$$

Note that the above bound for $x_{k+1}^{t_{i+1}}$ is also valid when $V_{2}\left(x_{k}^{t_{i+1}}\right)>1$. Therefore, it is concluded that the trajectories of system (3)-(4) are bounded, under constraints (33), (34), and (35), for all possible values of $V_{2}\left(x_{k}^{t_{i+1}}\right)$. Thus, stability in the impulse times is proven.

b) Boundedness between the impulses. From (20), disregarding the negative term $\rho_{i}\left(t_{i+1}\right) V_{2}\left(x_{k+1}^{t_{i+1}}\right)$, and recalling that $c_{1} c_{4}^{-\alpha_{1}} V_{2}^{\alpha_{1}}\left(x_{k}^{t_{i+1}}\right) \leq V_{1}\left(t_{i+1}, x_{k}^{t_{i+1}}\right)$; one gets

$$
\begin{aligned}
c_{1} c_{4}^{-\alpha_{1}} V_{2}^{\alpha_{1}}\left(x_{k}^{t_{i+1}}\right) \leq e^{-\beta T_{i}} V_{1}\left(t_{i}, x_{k}^{t_{i}}\right) & \\
& +\rho_{i}\left(t_{i+1}\right) \lambda V_{2}\left(x_{k}^{t_{i+1}}\right) .
\end{aligned}
$$

Once again, let us consider two cases for the boundedness proof, i.e. $V_{2}\left(x_{k}^{t_{i+1}}\right)>1$ and $V_{2}\left(x_{k}^{t_{i+1}}\right) \leq 1$. Then, from (37), it follows that for all $V_{2}\left(x_{k}^{t_{i+1}}\right)>1$

$$
V_{2}\left(x_{k}^{t_{i+1}}\right) \leq \frac{1}{\left(c_{1} c_{4}^{-\alpha_{1}}-\rho_{i}\left(t_{i+1}\right) \lambda\right)} V_{1}\left(t_{i}, x_{k}^{t_{i}}\right) .
$$

Note that $\rho_{i}\left(t_{i+1}\right) \lambda<c_{1} c_{4}^{-\alpha_{1}}$ has to hold in order to satisfy inequality (38). However, as it was previously described, if $c_{2} c_{4}^{\alpha_{1}-1}\left(c_{4}-c_{5}\right) \leq c_{1} c_{5}$ holds then $\rho_{i}\left(t_{i+1}\right) \lambda<c_{1} c_{4}^{-\alpha_{1}}$ is trivially satisfied, otherwise $T_{\max }<c_{2} \alpha / c_{5}$. Thus, applying (38) in (20) with the negative term $\rho_{i}(t) V_{2}\left(x_{k+1}^{t_{i+1}}\right)$ disregarding; recalling that $\rho_{i}\left(t_{i+1}\right) \lambda \leq c_{2} / c_{5}$, it is given that

$$
\begin{aligned}
V_{1}\left(t, x_{k}^{t}\right) & \leq\left(e^{-\beta\left(t-t_{i}\right)}+\frac{\rho_{i}\left(t_{i+1}\right) \lambda}{\left(c_{1} c_{4}^{-\alpha_{1}}-\rho_{i}\left(t_{i+1}\right) \lambda\right)}\right) V_{1}\left(t_{i}, x_{k}^{t_{i}}\right), \\
& \leq\left(\frac{c_{1} c_{5}+c_{2} c_{4}^{\alpha_{1}}}{c_{5}\left(c_{1}-\rho_{i}\left(t_{i+1}\right) \lambda c_{4}^{\alpha_{1}}\right)}\right) V_{1}\left(t_{i}, x_{k}^{t_{i}}\right) .
\end{aligned}
$$

Therefore, from (39), boundedness is obtained, i.e. $\left|x_{k}^{t}\right| \leq \sigma_{2}^{\prime}\left(\left|x_{k}^{t_{i}}\right|\right), \forall t \in\left[t_{i}, t_{i+1}\right)$, for $V_{2}\left(x_{k}^{t_{i+1}}\right)>1$ with

$$
\sigma_{2}^{\prime}\left(\left|x_{k}^{t_{i}}\right|\right)=\left(\frac{c_{2}\left(c_{1} c_{5}+c_{2} c_{4}^{a_{1} / b_{2}}\right)}{c_{1} c_{5}\left(c_{1}-\varrho \lambda c_{4}^{a_{1} / b_{2}}\right)}\left|x_{k}^{t_{i}}\right|^{a_{2}}\right)^{1 / a_{1}},
$$

where $\varrho=c_{2}\left(1-e^{-\gamma}\right) / c_{5}$, for all $i=k \in \mathbb{N}$. The boundedness proof is completed analyzing the case when $V_{2}\left(x_{k}^{t_{i+1}}\right) \leq 1$. From (37) it is obtained

$$
\begin{gathered}
c_{1} c_{4}^{-\alpha_{1}} V_{2}^{\alpha_{1}} x_{k}^{t_{i+1}} \leq e^{-\beta T_{i}} V_{1}\left(t_{i}, x_{k}^{t_{i}}\right)+\rho_{i}\left(t_{i+1}\right) \lambda, \\
V_{2}\left(x_{k}^{t_{i+1}}\right) \leq \frac{c_{4}}{c_{1}^{1 / \alpha_{1}}} V_{1}^{1 / \alpha_{1}}\left(t_{i}, x_{k}^{t_{i}}\right)+\frac{c_{2}^{1 / \alpha_{1}} c_{4}}{\left(c_{1} c_{5}\right)^{1 / \alpha_{1}}} .
\end{gathered}
$$

Thus, applying (40) in (20) with the negative term $\rho_{i}(t) V_{2}\left(x_{k+1}^{t_{i+1}}\right)$ disregarding, it follows that

$$
\begin{aligned}
V_{1}\left(t, x_{k}^{t}\right) & \leq e^{-\beta\left(t-t_{i}\right)} V_{1}\left(t_{i}, x_{k}^{t_{i}}\right) \\
& +\frac{c_{2} c_{4}}{c_{1}^{1 / \alpha_{1}} c_{5}} V_{1}^{1 / \alpha_{1}}\left(t_{i}, x_{k}^{t_{i}}\right)+\frac{c_{2}^{\alpha_{1}+1 / \alpha_{1}} c_{4}}{c_{1}^{1 / \alpha_{1}} c_{5}^{\alpha_{1}+1 / \alpha_{1}}} .
\end{aligned}
$$

Therefore, from (41), it is given that for all $i=k \in \mathbb{N}$, $\left|x_{k}^{t}\right| \leq \sigma_{2}\left(\left|x_{k}^{t_{i}}\right|\right)+\kappa_{2}, \forall t \in\left[t_{i}, t_{i+1}\right)$, for $V_{2}\left(x_{k}^{t_{i+1}}\right) \leq 1$ with

$$
\begin{gathered}
\sigma_{2}\left(\left|x_{k}^{t_{i}}\right|\right)=\left(\frac{c_{2}}{c_{1}}\left|x_{k}^{t_{i}}\right|^{a_{2}}+\frac{c}{c_{1}}\left|x_{k}^{t_{i}}\right|^{a_{2} b_{2} / a_{1}}\right)^{1 / a_{1}}, \\
c=c_{2}^{\left(a_{1}+b_{2}\right) / a_{1}} c_{4} / c_{1}^{b_{2} / a_{1}} c_{5}, \\
\kappa_{2}=\left(c_{2}^{\left(a_{1}+b_{2}\right) / a_{1}} c_{4} /\left(c_{1} c_{5}\right)^{\left(a_{1}+b_{2}\right) / a_{1}}\right)^{1 / a_{1}} .
\end{gathered}
$$

Thus, one can take the worst case between (39) and (41), and conclude that during each interval between impulses the trajectories of the system are bounded (Definition 1, eq. (10)) for every possible value of $V_{2}\left(x_{k}^{t_{i+1}}\right)$. Therefore, due to the stability property given by (9), the 2D system is $\mathrm{DS}$ in $\Omega$.

Remark 3 These results are local since $a_{1}>b_{2}$ and $b_{1}>a_{2}$, i.e. (33) and (35), respectively; only hold in a neighborhood of the origin, i.e. for all $i=k \in \mathbb{N},\left|x_{k}^{t}\right| \leq 1$ and $t \in\left[t_{i}, t_{i+1}\right)$.

Finally, let us consider the particular case in which $a_{1}=$ $b_{2}$ and $b_{1}=a_{2}$, i.e. $\alpha_{1}=1$ and $\beta_{1}=1$, respectively. Then, the following corollary is proposed.

Corollary 4 (Asymptotic diagonal stability - Maximum/Ranged dwell-time). Assume that there exist positive constants $\varepsilon \in(0,1), a_{1}, a_{2}, b_{1}, b_{2}, c_{1}, c_{2}, c_{3}, c_{4}$ and $c_{5}$ such that the vector Lyapunov function $V(\cdot)$ and its divergence along the trajectories of the system (3)-(4) satisfy (13), (14), (15), and the following constraints, for all $i=k \in \mathbb{N}, x_{k}^{t} \in \Omega$ and $t \in\left[t_{i}, t_{i+1}\right)$ 


$$
\begin{gathered}
c_{4}>c_{5} \wedge a_{1}=b_{2}, \\
c_{2}\left(c_{4}-c_{5}\right) \leq c_{1} c_{5} \vee T_{i}<\frac{c_{2}}{c_{5}} \alpha=T_{\max }, \\
b_{1}=a_{2} \wedge T_{\min }=\frac{c_{2}}{c_{5}} \gamma<T_{i},
\end{gathered}
$$

where $\gamma=-\ln \left[c_{3}(1-\varepsilon) /\left(c_{5}+c_{3}(1-\varepsilon)\right)\right]$, and

$$
\alpha=-\ln \left(\frac{c_{2}\left(c_{4}-c_{5}\right)-c_{1} c_{5}}{c_{2}\left(c_{4}-c_{5}\right)}\right) \text {, }
$$

for all $c_{2}\left(c_{4}-c_{5}\right)>c_{1} c_{5}$. Then, the $2 D$ system (3)-(4) is $A D S$ in $\Omega$ for any sequence $T_{i} \in\left[T_{\min }, T_{\max }\right]$.

Proof. Recalling that $c_{4}>c_{5}$, i.e. $\lambda \in(0,1)$; using (22) and (23) it follows that

$$
\begin{aligned}
\rho_{i}\left(t_{i+1}\right) V_{2}\left(x_{k+1}^{t_{i+1}}\right) \leq & \frac{c_{2} e^{-\beta T_{i}}}{c_{3}} V_{2}\left(x_{k}^{t_{i}}\right) \\
& \quad+\left(\rho_{i}\left(t_{i+1}\right) \lambda-c_{1} c_{4}^{-1}\right) V_{2}\left(x_{k}^{t_{i+1}}\right) .
\end{aligned}
$$

Then, as it was previously demonstrated, if $c_{2}\left(c_{4}-c_{5}\right) \leq$ $c_{1} c_{5}$ holds, then $\rho_{i}\left(t_{i+1}\right) \lambda<c_{1} c_{4}^{-1}$ is trivially satisfied, otherwise $T_{\max }<c_{2} \alpha / c_{5}$, with $\alpha$ defined as in Theorem 3 for $\alpha_{1}=1$; and then one obtains $V_{2}\left(x_{k+1}^{t_{i+1}}\right) \leq \varphi_{i}^{\prime} V_{2}\left(x_{k}^{t_{i}}\right)$, and by means of similar arguments as in Corollary 2, if $T_{\min }>c_{2} \gamma / c_{5}$, with $\gamma=-\ln \left[c_{3}(1-\varepsilon) /\left(c_{5}+c_{3}(1-\varepsilon)\right)\right]$; holds, then it is obtained that for all $i=k \in \mathbb{N}$

$$
\left|x_{k+1}^{t_{i+1}}\right| \leq \beta\left(\left|x_{0}\right|, k\right), \forall x_{0} \in \Omega,
$$

with $\beta\left(\left|x_{0}\right|, k\right)=c^{1 / b_{1}} \kappa^{(k+1) / b_{1}}\left|x_{0}\right|^{a_{1} / b_{1}}$, where $c=$ $c_{4} / c_{3}$, and $\kappa=c_{5} / c_{3}\left(1-e^{-\gamma}\right)<1$. Hence, the trajectories of system (3)-(4) converge to zero in the impulse times under constraints $T_{\min }>c_{2} \gamma / c_{5}, a_{1}=b_{2}, b_{1}=a_{2}$ and $\rho_{i}\left(t_{i+1}\right) \lambda<c_{1} c_{4}^{-1}$, if $c_{4}>c_{5}$ holds.

The boundedness between the impulses is proven as follows. From (37) it is given that

$$
c_{1} c_{4}^{-1} V_{2}\left(x_{k}^{t_{i+1}}\right) \leq e^{-\beta T_{i}} V_{1}\left(t_{i}, x_{k}^{t_{i}}\right)+\rho_{i}\left(t_{i+1}\right) \lambda V_{2}\left(x_{k}^{t_{i+1}}\right),
$$

and then, it follows that

$$
V_{2}\left(x_{k}^{t_{i+1}}\right) \leq \frac{1}{\left(c_{1} c_{4}^{-1}-\rho_{i}\left(t_{i+1}\right) \lambda\right)} V_{1}\left(t_{i}, x_{k}^{t_{i}}\right) .
$$

Thus, applying (46) in (20) with the negative term $\rho_{i}(t) V_{2}\left(x_{k+1}^{t_{i+1}}\right)$ disregarding, and recalling that $\rho_{i}\left(t_{i+1}\right) \lambda \leq$ $c_{2} / c_{5}$, it is given that

$$
V_{1}\left(t, x_{k}^{t}\right) \leq\left(\frac{c_{1} c_{5}+c_{2} c_{4}}{c_{5}\left(c_{1}-\rho_{i}\left(t_{i+1}\right) \lambda c_{4}\right)}\right) V_{1}\left(t_{i}, x_{k}^{t_{i}}\right) .
$$

Therefore, from (47), boundedness is obtained, i.e. $\left|x_{k}^{t}\right| \leq \sigma\left(\left|x_{k}^{t_{i}}\right|\right), \forall t \in\left[t_{i}, t_{i+1}\right)$, where

$$
\sigma\left(\left|x_{k}^{t_{i}}\right|\right)=\left(\frac{c_{2}\left(c_{1} c_{5}+c_{2} c_{4}\right)}{c_{1} c_{5}\left(c_{1}-\varrho \lambda c_{4}\right)}\right)^{1 / a_{1}}\left|x_{k}^{t_{i}}\right|^{a_{2} / a_{1}},
$$

with $\varrho=c_{2}\left(1-e^{-\gamma}\right) / c_{5}$, for all $i=k \in \mathbb{N}$. Hence, the trajectories of the system are bounded (Definition 2, eq. (8)), and due to the convergence property given by (7), the $2 \mathrm{D}$ system described by (3)-(4) is ADS in $\Omega$. Thus, the corollary has been proven.

Remark 4 Since $a_{1}=b_{2}$ and $b_{1}=a_{2}$, the results become global if (45) holds for all $x_{0} \in \mathbb{R}^{n}$.

Note that, from the statements given in Theorem 3 and Corollary 4 , the diagonal stability is straightforward for the case $a_{1}>b_{2}$ and $b_{1}=a_{2}$.

Contrary to the results given by [9] (Theorem 3.18, Proposition 3.24, Proposition 3.27) and [15], where asymptotic stability is obtained by means of a single Lyapunov function whose derivative is negative definite; the statements given in this work rely on a vector Lyapunov function. However, in our case the divergence operator, and not the derivative of a Lyapunov function, needs to satisfy condition (15), i.e. be negative definite. Particularly, the proposed results for minimal dwelltime are consistent with the ones in Proposition 3.24 (Persistent Flowing); while for maximum and ranged dwell-time with the ones in Proposition 3.27 (Persistent Jumping) in [9], respectively.

\section{LMI approach for a class of Lipschitz nonlin- ear impulsive systems}

In this section an LMI procedure to apply the main results is given to deal with impulsive systems containing linear terms but also Lipschitz nonlinearities.

Consider the following nonlinear impulsive system, consistent with (3)-(4), i.e.

$$
\begin{aligned}
\frac{d x_{k}^{t}}{d t} & =A x_{k}^{t}+\phi_{1}\left(x_{k}^{t}\right), \forall t \in\left[t_{i}, t_{i+1}\right), \\
x_{k+1}^{t} & =E x_{k}^{t}+\phi_{2}\left(x_{k}^{t}\right), \forall t=t_{i+1}, \forall i=k \in \mathbb{N},
\end{aligned}
$$

where $A \in \mathbb{R}^{n \times n}$ and $E \in \mathbb{R}^{n \times n}$ are constant matrices whilst $\phi_{1}\left(x_{k}^{t}\right): \Omega \rightarrow \mathbb{R}^{n}$ and $\phi_{2}\left(x_{k}^{t_{i+1}}\right): \Omega \rightarrow \mathbb{R}^{n}$ are Lipschitz nonlinearities for all $i=k \in \mathbb{N}$ and $t \in\left[t_{i}, t_{i+1}\right)$, i.e.

$$
\begin{aligned}
\left|\phi_{1}\left(x_{k}^{t}\right)-\phi_{1}\left(\bar{x}_{k}^{t}\right)\right| & \leq \psi_{1}\left|x_{k}^{t}-\bar{x}_{k}^{t}\right|, \\
\left|\phi_{2}\left(x_{k}^{t_{i+1}}\right)-\phi_{2}\left(\bar{x}_{k}^{t_{i+1}}\right)\right| & \leq \psi_{2}\left|x_{k}^{t_{i+1}}-\bar{x}_{k}^{t_{i+1}}\right|,
\end{aligned}
$$

where $\psi_{1}$ and $\psi_{2}$ are known positive constants for all $x_{k}^{t}, \bar{x}_{k}^{t} \in \Omega$. Define the vector Lyapunov function as follows

$$
V\left(t, x_{k}^{t}, x_{k+1}^{t_{i+1}}\right)=\left(\begin{array}{c}
x_{k}^{t^{T}} P_{1}(t) x_{k}^{t} \\
x_{k+1}^{t_{i+1}^{T}} P_{2}(k+1) x_{k+1}^{t_{i+1}}
\end{array}\right),
$$


where, inspired by [1] and [3], $P_{1}(t)=P_{11}+\frac{\left(T_{i}-\tau_{k}^{t}\right)}{T_{i}} P_{12}$ is a continuously differentiable with respect to $t$, symmetric, bounded, and positive definite matrix, with $d \tau_{k}^{t} / d t=1$, for all $t \in\left[t_{i}, t_{i+1}\right), \tau_{k+1}^{t_{i+1}}=0, P_{11}=P_{11}^{T}>0$ and $P_{12}=P_{12}^{T}>0$; while $P_{2}(p)=P_{21}+\tau_{p}^{t_{i+1}} P_{22}$ for $p=k, k+1$, are symmetric and positive definite matrices for all $i=k \in \mathbb{N}$, with $P_{21}=P_{21}^{T}>0$ and $P_{22}=P_{22}^{T}>0$.

Then, based on Corollary 2 (minimum dwell-time) or 4 (ranged dwell-time), the following result is stated.

Corollary 5 (LMI approach). Consider the vector Lyapunov function (50). If there exist matrices $P_{11}=P_{11}^{T}>$ $0, P_{12}=P_{12}^{T}>0, P_{21}=P_{21}^{T}>0$ and $P_{22}=P_{22}^{T}>0$, and a constant $c_{5}>0$ such that

$$
\begin{aligned}
& \left(\begin{array}{c|c}
-\mu_{1} I & P_{11}+P_{12} \\
\hline P_{11}+P_{12} & \Theta_{1}(\theta)
\end{array}\right) \leq 0, \\
& \left(\begin{array}{c|c}
-\mu_{1} I & P_{11} \\
\hline P_{11} & \Theta_{2}(\theta)
\end{array}\right) \leq 0, \quad\left(\begin{array}{c|c}
-\mu_{2} I & P_{21} E \\
\hline E^{T} P_{21} & \Upsilon(\theta)
\end{array}\right) \leq 0,
\end{aligned}
$$

with

$$
\begin{aligned}
& \Theta_{1}(\theta)=\left(P_{11}+P_{12}\right) A+A^{T}\left(P_{11}+P_{12}\right)-\theta^{-1} P_{12} \\
&+\left(\mu_{1} \psi_{1}^{2}+c_{5}\right) I \\
& \Theta_{2}(\theta)= P_{11} A+A^{T} P_{11}-\theta^{-1} P_{12}+\left(\mu_{1} \psi_{1}^{2}+c_{5}\right) I \\
& \Upsilon(\theta)=E^{T} P_{21} E-\left(P_{21}+\theta P_{22}\right) \\
&+\psi_{2}^{2}\left(\mu_{2} I+P_{21}+\theta P_{22}\right)+c_{5} I
\end{aligned}
$$

hold for all $\theta \in\left\{T_{\min }, T_{\max }\right\}$, as well as all the conditions of Corollary 2 (minimum dwell-time) or 4 (ranged dwell-time $)$, for $c_{1}=\lambda_{\min }\left(P_{11}\right), c_{2}=\lambda_{\max }\left(P_{11}+P_{12}\right)$, $c_{3}=\lambda_{\min }\left(P_{21}\right)$, and $c_{4}=\lambda_{\max }\left(P_{21}+\theta P_{22}\right)$; then, the Lipschitz nonlinear impulsive system (48)-(49) is SADS (Corollary 2) or ADS (Corollary 4). If $\phi_{1}$ and $\phi_{2}$ are globally Lipschitz, i.e. $\Omega=\mathbb{R}^{n}$; then, (48)-(49) is GSADS or $G A D S$

Proof. From divergence definition it is obtained that for all $t \in\left[t_{i}, t_{i+1}\right)$

$$
\begin{aligned}
\operatorname{div} V \leq & x_{k}^{t^{T}}\left[P_{1}(t) A+A^{T} P_{1}(t)+\frac{d P_{1}(t)}{d t}\right] x_{k}^{t} \\
& +x_{k}^{t_{i+1} T}\left[E^{T} P_{2}(k+1) E-P_{2}(k)\right] x_{k}^{t_{i+1}} \\
+ & 2 x_{k}^{t^{T}} P_{1}(t) \phi_{1}+2 x_{k}^{t_{i+1} T} E^{T} P_{2}(k+1) \phi_{2}+\phi_{2}^{T} P_{2}(k+1) \phi_{2} .
\end{aligned}
$$

Applying the well-known inequality $2 X^{T} Y \leq \mu^{-1} X^{T} X+$ $\mu Y^{T} Y$ for any scalar $\mu>0$, and taking into account that $\phi_{1}^{T} \phi_{1} \leq \psi_{1}^{2}\left|x_{k}^{t}\right|^{2}$ and $\phi_{2}^{T} \phi_{2} \leq \psi_{2}^{2}\left|x_{k}^{t_{i+1}}\right|^{2}$ hold due to the Lipschitz condition; it is given that $\operatorname{div} V$ is bounded as follows

$$
\operatorname{div} V \leq x_{k}^{t^{T}} \Xi_{1}(t) x_{k}^{t}+x_{k}^{t_{i+1} T} \Xi_{2}(k) x_{k}^{t_{i+1}},
$$

where

$$
\begin{gathered}
\Xi_{1}(t)=P_{1}(t) A+A^{T} P_{1}(t)+\frac{d P_{1}(t)}{d t}+\mu_{1}^{-1} P_{1}^{2}(t)+\mu_{1} \psi_{1}^{2} I \\
\Xi_{2}(k)=E^{T} P_{2}(k+1) E-P_{2}(k)+\mu_{2}^{-1} E^{T} P_{2}^{2}(k+1) E \\
+\psi_{2}^{2}\left(\mu_{2} I+P_{2}(k+1)\right)
\end{gathered}
$$

for any scalars $\mu_{1}, \mu_{2}>0$. Then, by Schur's complement, it follows that $\operatorname{div} V$ satisfies (15) if the following matrix inequality, as well as the right one in (52), holds, i.e.

$$
\left(\begin{array}{c|c}
-\mu_{1} I & P_{11}+\frac{\left(T_{i}-\tau_{k}^{t}\right)}{T_{i}} P_{12} \\
\hline P_{11}+\frac{\left(T_{i}-\tau_{k}^{t}\right)}{T_{i}} P_{12} & \Theta(t)
\end{array}\right) \leq 0,
$$

where

$$
\begin{aligned}
\Theta(t) & =P_{11} A+A^{T} P_{11} \\
& +\frac{\left(T_{i}-\tau_{k}^{t}\right)}{T_{i}}\left(P_{12} A+A^{T} P_{12}\right)-\frac{1}{T_{i}} P_{12}+\left(\mu_{1} \psi_{1}^{2}+c_{5}\right) I
\end{aligned}
$$

for all $\theta \in\left\{T_{\min }, T_{\max }\right\}$. Since (53) is affine in $\tau_{k}^{t}$, its negative definiteness is given by the negativeness over the finite set $\tau_{k}^{t} \in\left\{0, T_{i}\right\}$; hence, the LMI (51) and the left one in (52) are provided.

Hence, $\operatorname{div} V$ satisfies (15), for $a_{1}=b_{2}=2$ and $b_{1}=$ $a_{2}=2$, if the LMIs (51) and (52) hold for the finite set $\theta \in\left\{T_{\min }, T_{\max }\right\}$, with $c_{5}>0$.

Then, if all the conditions of Corollary 2 (minimum dwell-time) or 4 (ranged dwell-time) hold for $c_{1}=\lambda_{\min }\left(P_{11}\right), c_{2}=\lambda_{\max }\left(P_{11}+P_{12}\right), c_{3}=\lambda_{\min }\left(P_{21}\right)$, and $c_{4}=\lambda_{\max }\left(P_{21}+\theta P_{22}\right)$; the Lipschitz nonlinear impulsive system is SADS or ADS (GSADS or GADS if $\phi_{1}$ and $\phi_{2}$ are globally Lipschitz).

For the particular case of linear time-invariant systems, our method can be seen as a generalization of the result in [3]. One may remark that taking $P_{1}$ and $P_{2}$ in the same form for the statements given by Corollary 5 , leads to the conditions given by Theorem 2.2 (ranged dwelltime) and 2.3 (minimum dwell-time) in [3]. However, note that $P_{1}$ and $P_{2}$ are not independent with each other since they are coupled by means of the constants $c_{1}, c_{2}$, $c_{3}, c_{4}$ and $c_{5}$, which determine the stability and the type of dwell-time condition.

Note that Corollary 5 provides an easy way to deal with stability of Lipschitz nonlinear impulsive systems by means of simple quadratic Lyapunov functions and the $\Lambda$-inequality. In this sense, more complex tools like sumof-squares [3], convex characterizations [5], or loopedfunctional approach [6], may be applied to improve the application of our method as well as alternative inequalities to $\Lambda$-inequality [4]. 


\section{$5 \quad$ Simulation examples}

\subsection{Example 1: Globally Lipschitz nonlinearities}

Consider the nonlinear impulsive system (48)-(49) with

$$
\begin{gathered}
A=\left(\begin{array}{ll}
1 & 2 \\
1 & 3
\end{array}\right), E=\left(\begin{array}{cc}
-0.58 & 0.3 \\
0.4 & -0.68
\end{array}\right), \\
\phi_{1}\left(x_{k}^{t}\right)=\left(\begin{array}{c}
\sin \left(x_{1}^{t}\right) \\
0
\end{array}\right), \phi_{2}\left(x_{k}^{t}\right)=0.3\left(\begin{array}{c}
\sin \left(x_{1}^{t}\right) \\
\sin \left(x_{2}^{t}\right)
\end{array}\right) .
\end{gathered}
$$

and initial conditions $t_{0}=0, x_{0}=(2-2)^{T}$. Note that matrix $A$ is not Hurwitz whilst matrix $E$ is Schur, and the nonlinearities are globally Lipschitz.

Then, applying the statements given by Corollary 5 with $\mu_{1}=\mu_{2}=0.01, \psi_{1}=1$ and $\psi_{2}=0.3$, and using SeDuMi solver among YALMIP in MATLAB [20,13], a solution for the corresponding LMIs is founded, i.e.

$$
\begin{aligned}
& P_{11}=\left(\begin{array}{cc}
0.1152 & -0.0047 \\
-0.0047 & 0.1111
\end{array}\right), P_{12}=\left(\begin{array}{ll}
0.0483 & 0.0081 \\
0.0081 & 0.0599
\end{array}\right), \\
& P_{21}=\left(\begin{array}{ll}
0.3957 & 0.2217 \\
0.2217 & 0.2763
\end{array}\right), P_{22}=\left(\begin{array}{cc}
3.3851 & -3.1406 \\
-3.1406 & 4.6542
\end{array}\right),
\end{aligned}
$$

with $c_{1}=0.1080, c_{2}=0.1723, c_{3}=0.1064, c_{4}=0.5845$ and $c_{5}=0.0150$. Therefore, according to Corollary 4 and 5, the nonlinear impulsive system is GADS for all $0.1913>T_{i}>0$. It is easy to check that $c_{4}>c_{5}$ and $c_{2}\left(c_{4}-c_{5}\right)>c_{1} c_{5}$ hold, then the case for asymptotic diagonal stability is obtained. Some simulation results show that the system is unstable for $T_{i} \geq 0.2140$.

\subsection{Example 2: A more general case}

In order to depict the statements given by Theorem 3 , let us consider the scalar nonlinear impulsive system given by (3)-(4) with

$$
\begin{aligned}
f_{1}\left(x_{k}^{t}\right) & =x_{k}^{t^{3}}, \forall t \in\left[t_{i}, t_{i+1}\right), t_{0}=0 \\
f_{2}\left(x_{k+1}^{t}\right) & =0.8 x_{k}^{t^{2}}, \forall t=t_{i+1}, \forall i, k \in \mathbb{N}, x_{0}=0.5 .
\end{aligned}
$$

Note that in this case the nonlinear impulsive system does not have a particular structure as in Example 1. However, following similar arguments, it is still possible to provided some LMIs to prove the stability of the system.

Define the vector Lyapunov function as follows

$$
V\left(t, x_{k}^{t}, x_{k+1}^{t_{i+1}}\right)=\left(\begin{array}{c}
\frac{1}{2} x_{k}^{t^{2}}+\frac{\left(T_{i}-\tau_{k}^{t}\right) p_{1}}{2 T_{i}} x_{k}^{t^{2}} \\
x_{k+1}^{t_{i+1} \frac{1}{2}}
\end{array}\right),
$$

where $p_{1}>0$ is a positive constant and $d \tau_{k}^{t} / d t=1$, for all $t \in\left[t_{i}, t_{i+1}\right), \tau_{k+1}^{t_{i+1}}=0$. Note that $x_{k}^{t_{i+1} \frac{1}{2}}$ is welldefined for all $x_{0} \geq 0$. Let us consider the local case in which the trajectory satisfies $\left|x_{k}^{t}\right| \leq 1$. Then, it is easy to check that for all $\left|x_{k}^{t}\right| \leq 1$, the following inequalities hold:

$$
\begin{gathered}
c_{1}\left|x_{k}^{t}\right|^{4} \leq V_{1}\left(t, x_{k}^{t}\right) \leq c_{2}\left|x_{k}^{t}\right|^{\frac{1}{2}}, \\
c_{3}\left|x_{k}^{p}\right| \leq V_{2}\left(x_{k}^{p}\right) \leq c_{4}\left|x_{k}^{p}\right|^{\frac{1}{2}}, \forall p=t_{i}, t_{i+1},
\end{gathered}
$$

with $c_{1}=1 / 2, c_{2}=\left(p_{1}+1\right) / 2, c_{3}=1$ and $c_{4}=1$. From divergence definition it is obtained that for all $t \in$ $\left[t_{i}, t_{i+1}\right)$

$$
\operatorname{div} V \leq\left(\frac{T_{i}+\left(T_{i}-\tau_{k}^{t}\right) p_{1}}{T_{i}}-\frac{p_{1}}{2 T_{i}}\right) x_{k}^{t^{2}}-0.1056 x_{k}^{t_{i+1}{ }^{3}}
$$

Therefore, $\operatorname{div} V$ will satisfy (15), for the case $a_{1}>b_{2}$, $b_{1}>a_{2}$ with $a_{1}=4, a_{2}=1 / 2, b_{1}=1$ and $b_{2}=1 / 2$ (Theorem 3); if the following LMI holds

$$
\left(\begin{array}{c|c}
\frac{T_{i}+\left(T_{i}-\tau_{k}^{t}\right) p_{1}}{T_{i}}-\frac{p_{1}}{2 T_{i}}+c_{5} & 0 \\
\hline 0 & -0.1056+c_{5}
\end{array}\right) \leq 0 .
$$

Hence, since previous inequality is affine in $\tau_{k}^{t}$, the following LMIs are provided

$$
\begin{gathered}
\left(\begin{array}{c|c}
1+p_{1}-\frac{p_{1}}{2 \theta}+c_{5} & 0 \\
\hline 0 & -0.1056+c_{5}
\end{array}\right) \leq 0, \\
\left(\begin{array}{c|c}
1-\frac{p_{1}}{2 \theta}+c_{5} & 0 \\
\hline 0 & -0.1056+c_{5}
\end{array}\right) \leq 0,
\end{gathered}
$$

where $\theta \in\left\{T_{\min }, T_{\max }\right\}$. Thus, it is concluded that $\operatorname{div} V$ satisfies (15), for the conditions of Theorem 3, if the previous LMIs hold for the finite set $\theta \in\left\{T_{\min }, T_{\max }\right\}$, with $c_{5}>0$.

Then, using SeDuMi among YALMIP, the following solution for the LMIs is founded: $p_{1}=0.4247$ and $c_{5}=$ 0.0528. Thus, according to Theorem 3 , the nonlinear impulsive system is locally DS for all $1.0384>T_{i}>0$. It is easy to check that $c_{4}>c_{5}$ and $c_{2} c_{4}^{3}\left(c_{4}-c_{5}\right)>c_{1} c_{5}$ hold, thus the case for local diagonal stability is obtained. Some simulation results show that the system is unstable for $T_{i} \geq 1.2001$ which gives an idea about the conservatism of our results.

According to these results one may remark for both examples that, although only sufficient conditions are provided, the estimate of the maximum reset interval is not that far from the instability bound. From this point of view, the method provides an interesting trade off between accuracy of the analysis and simplicity. 


\section{Conclusions}

In this paper the stability notion for a class of nonlinear impulsive systems is studied using a $2 \mathrm{D}$ vector Lyapunov function approach. The given methodology contributes to the development of a new stability analysis for nonlinear impulsive dynamical systems based on a vector Lyapunov function and its divergence operator in a 2D time domain. Different types of stability notions for a class of nonlinear impulsive systems are studied using this vector Lyapunov function approach. The results are applied to analyze the stability of a class of Lipschitz nonlinear impulsive systems based on LMIs, and some numerical results illustrate the feasibility of the proposed approach.

\section{References}

[1] L.I Allerhand and U. Shaked. Robust stability and stabilization of linear switched systems with dwell time. IEEE Transactions on Automatic Control, 56(2):381-386, 2011.

[2] D. Bainov and P. Simeonov. Systems with Impulse Effects: Stability, Theory and Applications. Academy Press, 1989.

[3] C. Briat. Convex conditions for robust stability analysis and stabilization of linear aperiodic impulsive and sampled-data systems under dwell-time constraints. Automatica, 49:3449$3457,2013$.

[4] C. Briat. Linear Parameter-Varying and Time-Delay Systems - Analysis, Observation, Filtering \& Control, volume 3 of Advances on Delays and Dynamics. SpringerVerlag, Heidelberg, Germany, 2015.

[5] C. Briat and A. Seuret. Convex dwell-time characterizations for uncertain linear impulsive systems. IEEE Transactions on Automatic Control, 57(12):3241-3246, 2012.

[6] C. Briat and A. Seuret. A looped-functional approach for robust stability of linear impulsive systems. Systems 8 Control Letters, 61(10):980-988, 2012.

[7] M. Emelianov, P. Pakshin, K. Gałkowski, and E. Rogers. Stability and stabilization of differential nonlinear repetitive processes with applications. In Proceedings of the 19th IFAC World Congress, pages 5467-5472, Cape Town, South Africa, 2014.

[8] J. Emelianova, P. Pakshin, K. Gałkowski, and E. Rogers. Vector Lyapunov function based stability of a class of applications relevant $2 \mathrm{~d}$ nonlinear systems. In Proceedings of the 19th IFAC World Congress, pages 8247-8252, Cape Town, South Africa, 2014.

[9] R. Goebel, R.G. Sanfelice, and A.R. Teel. Hybrid dynamical systems. IEEE Control Systems Magazine, 29(2):28-93, 2009.

[10] L. Hu, Y. Cao, and H. Shao. Constrained robust sampleddata control of nonlinear uncertain systems. International Journal of Robust and Nonlinear Control, 12:447-155, 2002.

[11] V. Lakshmikantham, V. M. Matrosov, and S. Sivasundaram. Vector Lyapunov Functions and Stability Analysis of Nonlinear Systems. Mathematics and its Applications. Kluwer Academic Publishers Group, Dordrecht, 1991.

[12] L. Lee, Y. Liu, J. Liang, and X. Cai. Finite time stability of nonlinear impulsive systems and its applications in sampled-data systems. ISA Transactions, 2015. DOI:10.1016/j.isatra.2015.02.001.
[13] J. Löfberg. Yalmip: A toolbox for modeling and optimization in MATLAB. In Proceedings of the CACSD Conference, Taipei, Taiwan, 2004.

[14] P. Naghshtabrizi, J.P. Hespanha, and A.R. Teel. Exponential stability of impulsive systems with application to uncertain sampled-data systems. Systems and Control Letters, 57(5):378-385, 2008.

[15] D. Nešić, A.R. Teel, and L. Zaccarian. Stability and performance of siso control systems with first-order reset elements. IEEE Transactions on Automatic Control, 56(11):2567-2582, 2011.

[16] H. Ríos, L. Hetel, and D. Efimov. Vector Lyapunov function based stability for a class of impulsive systems. In Proceedings of the 54th IEEE Conference on Decision and Control, pages 2247-2251, Osaka, Japan, 2015.

[17] E. Rogers, K. Galkowski, and D. Owens. Control Systems Theory and Applications for Linear Repetitive Processes, volume 349 of Lecture Notes in Control and Information Sciences. Springer-Verlag, Berlin, 2007.

[18] A. Seuret. A novel stability analysis of linear systems under asynchronous samplings. Automatica, 48(1):177-182, 2012.

[19] N. Sivashankar and P.P. Khargonekar. Characterization of the $\mathcal{L}_{2}$-induced norm for linear systems with jumps with applications to sampled-data systems. SIAM Journal on Control and Optimization, 32(4):1128-1150, 1994.

[20] J.F. Sturm. Using SEDUMI 1.02, a MATLAB toolbox for optimization over symmetric cones. Optimization Methods and Software, 11(12):625-653, 2001.

[21] H. $\mathrm{Xu}$ and K.L. Teo. Exponential stability with $\mathcal{L}_{2}$-gain condition of nonlinear impulsive switched systems. IEEE Transactions on Automatic Control, 55(10):2429-2433, 2010.

[22] N. Yeganefar, N. Yeganefar, M. Ghamgui, and E. Moulay. Lyapunov theory for 2-D nonlinear roesser models: Application to asymptotic and exponential stability. IEEE Transactions on Automatic Control, 58(5):1299-1304, 2013.

[23] S.T. Zavalishchin and A.N. Sesekin. Dynamic Impulse Systems. Theory and Applications. Mathematics and its Applications. Kluwer Academic Publishers Group, Dordrecht, 1997.

[24] Q. Zhu and G.-D. Hu. Stability and absolute stability of a general 2-d non-linear fm second model. IET Control Theory E Applications, 5(1):239-246, 2011.

[25] Q. Zhu, G.-D. Hu, and Y.-X. Yin. Lyapunov-type theorem of general two-dimensional nonlinear parameter-varying fm second model. IEEE Transactions on Circuits and Systems II: Express Briefs, 59(7):453-457, 2012. 